$\mathrm{KE}$, eds. Die Normale und die Gestörte Pupillenbewegung. München: Bergman JF, 1973:194-8.

6 Wagener HP. Enophthalmos in Horner's syndrome. Am J Ophthalmol 1934;17:209-14.

7 Lepore FL. Enophthalmos and Horner's syndrome. Arch Neurol 1983;40:460.

8 Nielsen PJ. Upside down ptosis in patients with Horner's syndrome. Acta Ophthalmol 1983;61:952-7.

Accepted 17 January 1986

\section{Aphemia as a first symptom of multiple sclerosis}

Sir: Speech disorders are common in the course of multiple sclerosis, but aphasia is rare. $^{1-3}$ We describe a patient with aphemia, that is, an articulation disorder caused by a left hemisphere lesion, as a first symptom of multiple sclerosis.

A 29 year old right handed female medical student complained of speech problems and of a loss of strength in the right hand, which had developed insidiously in 5 days. The speech difficulties mainly consisted of an altered inflection. Writing was unimpaired, but less skilfully performed. She had no other symptoms and her medical history was uneventful. Apart from contraceptives no medication was taken. Neurological examination disclosed a minimal weakness of the right arm and leg. Cranial nerves, coordination, sensory testing, and reflexes were normal, with both plantar responses flexor. There were no signs of bucco-facial apraxia. There was no dysphagia, increase of facial reflexes or lability of emotional expression. Her speech was monotonous with a loss of interpunction and emotional expression. Neuropsychological examination did not show any language disorder. Conversational speech was fluent with a normal syntax and vocabulary, without paraphasias. Comprehension of spoken and written language was excellent. Confrontation naming, series speech, reading aloud, writing, and repetition of spoken language were entirely normal. No ideational or ideomotor apraxia was present. Visuospatial skills, tactile perception and memory functions were unimpaired. There was no attentional or planning disturbance.

The speech difficulty was characterised by hesitation in starting a sentence and in pronouncing words starting with a consonant. She did not simplify certain phonemes, but rather gave the impression of stuttering although there was no actual repetition of phonemes. The speech difficulty was not constant for a certain word or sound, but changed during conversation. Inflection was radically altered. She could not sing a scale or simple songs because of the dysprosody, but humming was normal. The patient was definitely concerned about her symptoms.

Extensive blood examination disclosed no abnormality. There was no serological evidence of viral or treponemal infection. A contrast enhanced CT scan of the brain on admission was normal. Cerebrospinal fluid (CSF) contained 73 erythrocytes, 16 lymphocytes, and 2 monocytes per $\mathrm{mm}^{3}$. Total protein was $0.35 \mathrm{~g} / \mathrm{l}$ with an IgG index of 1.58 (normal less than 0.60 ) and a normal albumin ratio. Electrophoresis showed oligoclonal bands confirming intrathecal IgG synthesis EEG and evoked potential studies (visual, sensory and auditory) were normal. Within a few days the weakness disappeared and in 2 weeks there was a resolution of the speech disorder. A second CT scan now showed a hypodense area with central contrast enhancement in the frontal white matter of the left hemisphere (fig a). She was discharged in good condition and resumed her studies without signs of recurrent neurological deficit during a follow-up of one year. After 6 months magnetic resonance imaging (MRI) showed multiple paraventricular lesions (fig b). Spontaneous recovery, CSF findings, and lack of any other explanation were suggestive of multiple sclerosis. In combination with the MRI findings 6 months later the criteria for laboratory supported definite multiple sclerosis were fulfilled. ${ }^{45}$

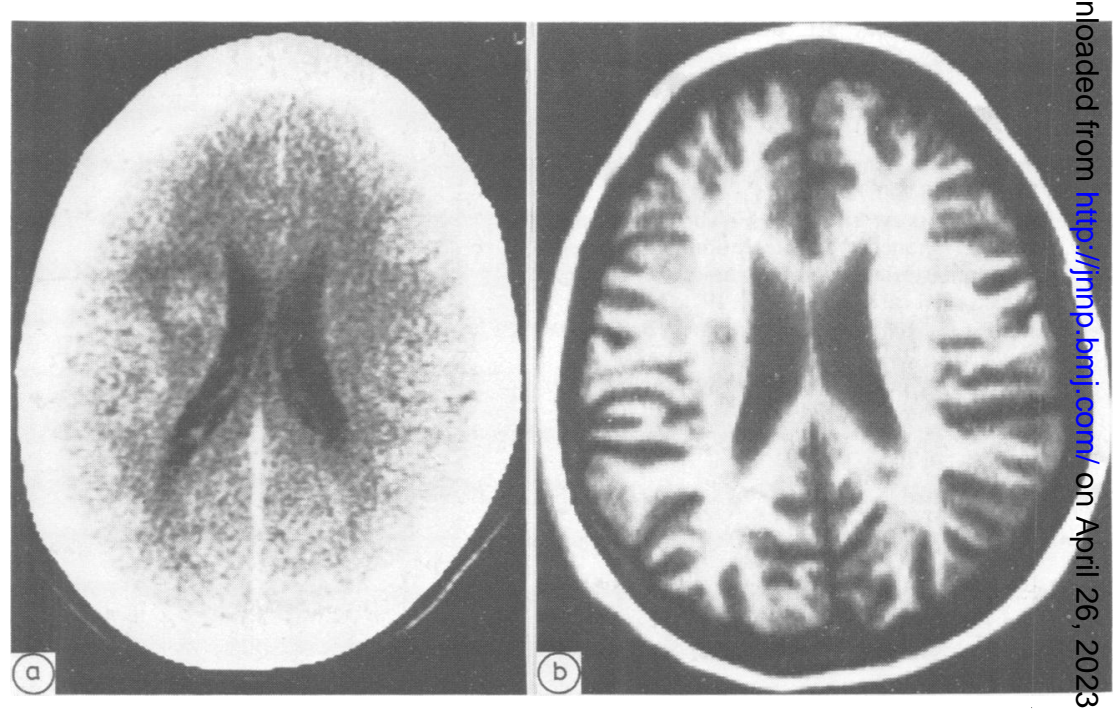

Fig (a) Contrast enhanced CT scan at the level of the cella media, (b) axial MRI scan, 6 ত months later, at the level of the cella media. (TR $1400 \mathrm{~ms}$; TE $32 \mathrm{~ms} ;$ TI $400 \mathrm{~ms}$ ).
Abnormal speech with normal propos tional language can be subdivided into three groups according to the kind of articulators disorder and the accompanying neurological symptoms and signs: dysarthria, aprosodia and aphemia. In dysarthria the articulation disorder is basically a simplification of arties ulation with articulatory distortions. ${ }^{6}$ In the aprosodies the affective components of speech are impaired after right hemisphere lesions. ${ }^{7}$ The third group, called aphemia of apraxia of speech comprises articulation disorders which have caused much debate Different authors have stressed aphasie dysarthric, or apraxic aspects, and namesp this group of articulation disorders accorod ingly. ${ }^{8}$ Basically aphemia concerns in volvement of a purely expressive aspect ớ spoken language, and as such it is difficult t $\vec{\oplus}$ distinguish from motor aphasia. ${ }^{9}$ Propositional language however is entirely norma $\vec{\omega}$ There has been discussion about the intes pretation of phonematic errors as aphasic of apraxic. ${ }^{10}$ The inconsistency of the articto latory problems is used as a distinction frorg dysarthria and to stress the apraxic aspect. A frequent concomitant finding is oral or facial apraxia. ${ }^{11}$ The symptoms of oub patient fit in with the picture of aphemia. left-sided lesion of the posterior part of the inferior frontal lobe and especially the oee $\vec{B}$ white matter of the anterior limb of dhe internal capsule is the most consisent pathological finding. The aetiology is near. $\vec{b}$ always vascular or traumatic. ${ }^{8}$ In Dup patient a lesion became visible on the scan which extended into the area of the 
putamen and the anterior limb of the internal capsula. The latter localisation could be responsible for the aphemia. To our knowledge aphemia has not been described previously as a first symptom of multiple sclerosis.

We thank Prof Dr J Valk (MRI scan) and Dr B Verbeeten jr (CT scan). We also thank $\mathrm{R}$ Bierau for secretarial assistance.

D HERDERSCHEÊ J STAM

MMA DERIX

Department of Neurology, Academisch Medisch Centrum, 1105 AZ Amsterdam, The Netherlands

\section{References}

1 Benson DF. Aphasia, Alexia and Agraphia. Clinical Neurology and Neurosurgery Monographs. Churchill Livingstone, 1979.

2 Farmakides MN, Boone DR. Speech problems of patients with multiple sclerosis. $J$ Speech Hear Disorder 1960;25:385-90.

3 Olmos-Lau N, Ginsberg MD. Aphasia in multiple sclerosis. Neurology 1977;27:623-6.

4 Poser CM, Paty DW, Scheinberg L, et al. New diagnostic criteria for multiple sclerosis: Guidelines for research protocols. Ann Neurol 1983;13:227-31.

5 Gebarski SS, Gabrielsen TO, Gilman S, Knake JE, Latack JT, Aisen AM. The initial diagnosis of multiple sclerosis: clinical impact of magnetic resonance imaging. Ann Neurol 1985;17:469-74.

6 Johns DF, Darley FL. Phonemic variability in apraxia of speech. J Speech Hear Res 1970; 13:556-83.

7 Ross ED, Harney JH, deLacoste-Utamsing Ch, Purdy PD. How the brain integrates affective and propositional language into a unified behavioral function. Arch Neurol 1981;38:745-8.

8 Schiff HB, Alexander MP. Aphemia. Arch Neurol 1983;40:720-7.

9 Roch Lecours A, Lhermitte F. The pure form of the phonetic desintegration syndrome (pure anarthria); anatomo-clinical report of a historical case. Brain Lang 1976;3:88-113.

10 Darley FL. Aphasia Philadelphia: WB Saunders, 1982.

11 Nathan PW. Facial apraxia and apraxic dysarthria. Brain 1947;70:449-78.

Accepted I August 1986

\section{Limb girdle type muscular dystrophy associ-} ated with a Wolf-Parkinson-White syndrome

Sir: Cardiac involvement varies greatly with different types of muscular dystrophy. In general the so called dystrophic heart disease is common in Duchenne's muscular dystrophy $(50-80 \%)$, infrequent in limb girdle type muscular dystrophy ${ }^{1}$ and occasional in all other types of muscular dystrophy. The most important clinical features of myocardial disease in muscular dystrophies are tachycardias, ${ }^{1}$ arrhythmias, ${ }^{23}$ congestive heart failure and sudden death. ${ }^{4}$ Various publications emphasise the even more frequent appearance of an altered ECG. ${ }^{15}$ The Wolff-Parkinson-White syndrome has been described in patients with cardiomyopathy ${ }^{210}$ and in three cases with Duchenne's muscular dystrophy. ${ }^{7-9}$ To our knowledge the syndrome has not been reported in association with limb girdle type muscular dystrophy.

A 40 year old male patient was admitted because of weakness and atrophy of shoulder and pelvic girdle muscles. He had a history of 12 years with lumbosacral pain and slow deterioration of muscular function which incapacitated the patient in his daily activities. Examination revealed normal vital signs, a weight of $45 \mathrm{~kg}$ and a height of $168 \mathrm{~cm}$. Cardiologic examination was normal. The patient had severe symmetrical proximal muscular atrophy of both upper and lower limbs. Distal, facial and abdominal muscles were not affected, and there were no skeletal deformities. Biceps, triceps and knee reflexes were diminished. The patient had a lordotic gait, difficulty in climbing stairs, combing his hair and Gower's sign was positive. The rest of the neurological examination was normal.

Laboratory data revealed a CK of $500 \mathrm{U}$
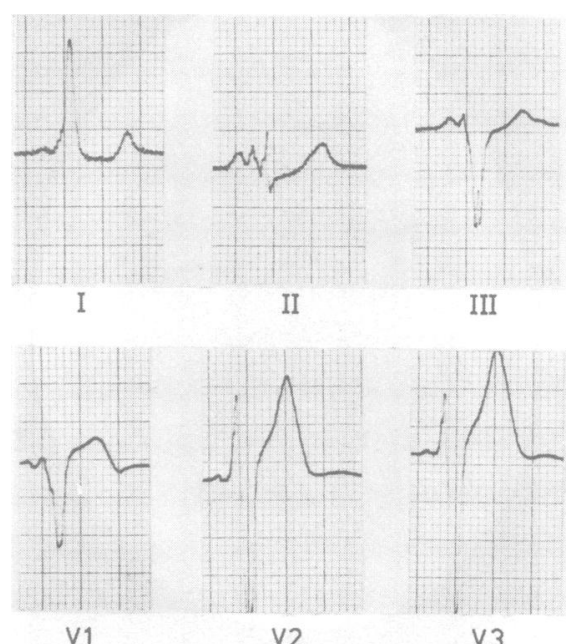

V1

V2

V3

Fig Electrocardiogram demonstrating a prolonged $Q R S$ complex shortened $Q T$ and $P J$ times and delta waves. (normal up to $170 \mathrm{U}$ ) with a muscle fraction of $1 \cdot 1 \%$, LDH, SGOT and all other standard laboratory tests were normal. Chest and abdominal radiographs were normal. The resting ECG showed a sinus bradycardia of 49 beats per minute and an axis of $-20^{\circ}$. The QRS complex measured $0.15 \mathrm{~ms}$, the PR interval $0.1 \mathrm{~ms}$ and the PJ interval $0.26 \mathrm{~ms}$. Positive delta waves were seen in leads I, aVL and V4-V6 and a negative delta wave was observed in V1 compatible with a type B Wolff-ParkinsonWhite syndrome (fig). The ECG also revealed sinus bradycardia which was reversed by exercise and thought to be physiological. An M-mode, two dimensional and dynamic echocardiogram did not reveal abnormalities and all standard measurements were normal. The EMG showed fibrillations of low amplitude and polyphasic motor deltoid, biceps, quadriceps and anterior tibial muscles. Neuroconduction was normal. The muscular biopsy specimen showed muscle fibre necrosis, wide variations in fibre size, increased connective tissue and internal muscle fibre nuclei, being compatible with muscular dystrophy. We have obtained detailed information of 38 family members. One brother of the patient was found to have the same progressive muscular process but withou cardiac involvement. He was 9 years olden than our patient and his disease hat progressed further. A muscle biopsy showe $\vec{c}$ the same myopathic features.

Limb girdle type muscular dystrophy is a $\vec{F}$ autosomal recessive disorder characterised by insidious onset in early adulthood and

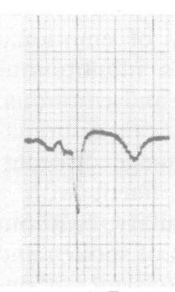

aVR
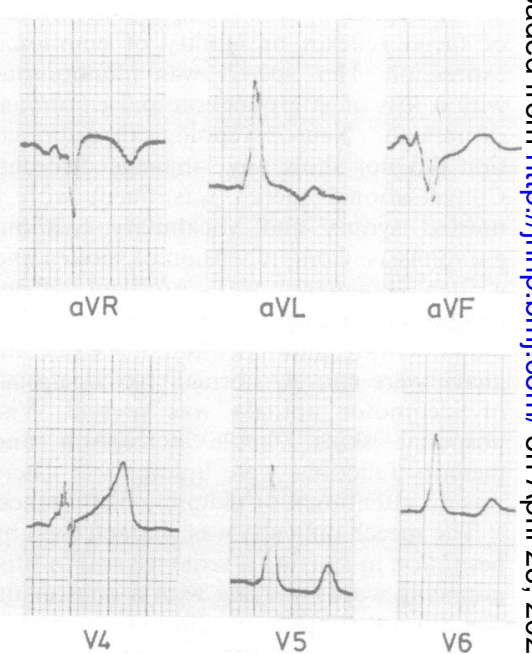

V6 